\title{
Effects of supplementing n-3 fatty acid enriched eggs and walnuts on cardiovascular disease risk markers in healthy free-living lacto-ovo-vegetarians: a randomized, crossover, free-living intervention study
}

\author{
Bonny Burns-Whitmore ${ }^{1,2}$, Ella Haddad ${ }^{1}$, Joan Sabaté ${ }^{1}$ and Sujatha Rajaram ${ }^{1 *}$
}

\begin{abstract}
Background: Plant and marine n-3 fatty acids (FA) may favorably modify select markers of cardiovascular disease risk. Whether supplementing the habitual diet of lacto-ovo-vegetarians (LOV) with walnuts (containing a-linolenic acid, ALA) and n-3 FA enriched eggs (containing primarily docosahexaenoic acid, DHA and ALA) would have equivalent effects on CVD risk factors is explored in this study.

Methods: In this study, 20 healthy free-living LOVs following their habitual diet were randomly assigned in a crossover design to receive one of three supplements: n-3 FA enriched egg (6/week), walnuts ( $28.4 \mathrm{~g}, 6 /$ week) or a standard egg, 6/week (control) for 8 weeks each with 4-wk washout between treatments. Erythrocyte membrane fatty acids, serum lipids and inflammatory markers were measured th the end of each treatment.

Results: Dietary compliance was observed by an expected increase in erythrocyte membrane ALA following the walnut treatment and in DHA following the $n-3$ FA enriched egg treatment. Walnut treatment lowered serum triacylglycerol, total cholesterol and Apo B $(p<0.05)$ compared to the standard egg but not the $n-3$ FA enriched egg treatment. However, walnut treatment significantly reduced total: HDL cholesterol ratio compared to both egg treatments. There were no differences between treatments for any of the inflammatory markers.
\end{abstract}

Conclusions: For LOV, a direct source of DHA such as n-3 FA enriched eggs seems necessary to increase membrane levels of DHA. However for producing an overall favorable blood lipid profile, daily consumption of a handful of walnuts rich in ALA may be a preferred option for lacto-ovo vegetarian.

Keywords: Walnuts, n-3 fatty acid enriched eggs, Lacto-ovo vegetarians, Cardiovascular risk factors, Eggs, Inflammation markers, Blood lipids

\section{Background}

The major plant n-3 fatty acid $\alpha$-linolenic acid (ALA) is found in walnuts, soybean, canola oil, and flaxseed; while the major marine $\mathrm{n}-3$ fatty acids, eicosapentaenoic (EPA) and docosahexaenoic acids (DHA), are found in coldwater fatty fish and flax or soy-fed poultry and farm animals $[1,2]$. Diets containing high amounts of both plant

\footnotetext{
* Correspondence: srajaram@llu.edu

'Department of Nutrition, School of Public Health, Loma Linda University, Loma Linda, CA 92350, USA

Full list of author information is available at the end of the article
}

and marine derived n-3 fatty acids are associated with reduced risk of cardiovascular disease (CVD) [3,4], but studies indicate Americans do not consume enough n-3 fatty acids $[1,5,6]$. The cardio-protective effects of $n-3$ fatty acids may be partially mediated via favorable changes in blood lipids and inflammation $[7,8]$. Whether or not ALA and EPA/DHA have equivalent effects on CVD risk factors specifically in healthy lacto-ovo-vegetarians (LOV) has not extensively studied.

Some fortified dairy products and eggs contain very small amounts of DHA (0.02-0.3 g/serving) and are the 
only direct sources of DHA in the LOV diet $[1,6]$. The only vegan DHA option is an algae-based supplement [9]. Vegans and LOVs depend on the endogenous production of EPA and DHA from ALA; however, this endogenous conversion is inefficient with only $5-7 \%$ of dietary ALA converting to EPA and DHA [10]. The dietary ratio of $n-6$ to $n-3$ fatty acids influences conversion of ALA to EPA and DHA [6]. While LOVs and vegans do consume significant amount of ALA in their diet, overall their linoleic acid (LA) intake is many folds higher than ALA intake producing high n-6: n-3 fatty acid ratio. This may suppress the conversion of ALA to EPA and DHA $(1,6)$.

Consumption of 57-71 g/day of ALA rich walnuts decreases total and LDL cholesterol (LDL-C) and triacylglycerol (TAG) in normo- and hypercholesterolemic individuals, resulting in a favorable reduction in CVD risk (11-16). Studies with cold-water fatty fish demonstrate reduction of atherosclerotic plaque formation, lowering of TAG and an increase in HDL-C, supporting the rationale for including fatty fish in the diet to prevent Coronary Heart Disease [3,11-13]. We recently examined the effects of consuming fatty fish and walnuts on serum lipids and found that walnuts lowered total and LDL-C while the fatty fish decreased serum TAG and increased HDL-C [14]. Since LOVs do not consume fish, an alternate source of long chain n-3 fatty acids for LOVs could be n-3 fatty acid enriched eggs. Chickens fed flaxseed, soy, or algal marine sources, incorporate DHA and ALA into their eggs, which results in the production of n-3 fatty acid enriched eggs $[15,16]$.

N-3 fatty acid enriched eggs fed to hypercholesterolemic non-vegetarians showed increase in HDL-C, decrease in platelet aggregation, and significant lowering of TAG [17-20]. Some studies have looked at the effect of feeding n-3 fatty acid enriched eggs on blood lipids, but none have compared the effects of ALA-rich walnuts with that of $n-3$ fatty acid (primarily DHA) enriched egg on CVD risk factors especially among LOVs.

\section{Methods}

\section{Participants}

Healthy LOV subjects were recruited from southern California via a multistage screening process with community advertisements through local newspapers and flyers. Potential candidates that responded were presented with the study information at group meetings and were asked to sign the informed consent if they decided to proceed with the study. Screening process also included interviews with two investigators to assess potential compliance. Men and women between the ages of 21-90 years and who had normal total cholesterol (Total-C) and TAG levels were included in the study. Participants were excluded if they had existing medical conditions, were $<21$ or $\geq 90$ years of age, were smokers, drank alcohol, took supplements, not willing to follow dietary intervention and instructions, were on cholesterol lowering medications, or on daily prescription antiinflammatory medication, and were LOV for $<3$ months. The minimum sample size required for a crossover design to detect a mean absolute difference in serum LDL-C of $0.26 \mathrm{mmol} / \mathrm{L}$ at $\alpha=0.05$ and power $>80 \%$ was calculated to be 18 . Assuming a dropout rate of $20 \%$, we recruited 26 subjects for this study. All participants signed a written informed consent approved by the Internal Review Boards at Loma Linda University, Loma Linda, CA and California State Polytechnic University, Pomona, CA.

\section{Study design and protocol}

The study was a randomized, crossover $(3 \times 3$ Latinsquare), free-living intervention study in which subjects were blinded to the egg treatments. Participants were asked to follow their habitual diet and add the treatment food as a supplement. They were randomly assigned to n-3 fatty acid enriched egg (6/wk), walnuts (28.4 g, 6x/wk) or standard egg, 6/wk (control). Treatments were followed for 8 weeks each in a crossover design, with a 4-week washout period between treatments. Previous studies $[14,21,22]$ have shown that 3-6 weeks intervention and 4 weeks washout is adequate to see changes especially for serum lipids and red cell membrane fatty acids.

The n-3 fatty acid enriched eggs were obtained from chickens fed an organic, vegetarian diet containing flaxseed and the standard eggs for the control treatment were obtained from chickens fed organic, vegetarian diet. The $\mathrm{n}-3$ fatty acid composition of the two eggs was previously published [23]. The $\mathrm{n}-3$ fatty acid enriched egg contained $500 \mathrm{mg}$ DHA, $40 \mathrm{mg}$ EPA and $1 \mathrm{~g}$ ALA per egg yolk while the standard egg yolk contained $110 \mathrm{mg}$ DHA, negligible amount of EPA and $0.15 \mathrm{~g}$ ALA. The walnuts used in the study provided $2.95 \mathrm{~g}$ ALA and 10.4 LA per 28.4 grams or 1 ounce of walnuts (Covance, Madison, WI).

Participants received diet counseling and their intervention foods from a Registered Dietitian (RD) every two weeks during the treatment periods. Participants were asked to avoid excess amounts of foods rich in n-3 fatty acids such as flax, soy, or canola oil, and to refrain from consuming any additional eggs or walnuts other than the ones supplied during the study. The instructions provided to the subjects were to add the treatment food to their habitual diet. They could incorporate the foods in any form they desired. Eggs could be cooked as omelet or hard boiled eggs or incorporated into a dish provided the participant consumed the entire serving. Walnuts could be eaten raw or used on salads, muffins, etc. Diet and caloric consumption was determined by 
doing 24-hour recalls. This was a free-living study, so there were no instructions provided regarding caloric intake. Subjects were asked to maintain the same lifestyle habits with which they entered the study such as the level and type of physical activity.

\section{Quality control}

Quality control and compliance was ensured among study participants by: 1) counseling with the RD throughout the study; 2) maintaining a daily diary to record deviations in food and medicine intake; 3) obtaining three 24-hour recalls during each treatment period to assess nutrient intake (Nutrition Data System-Research software -NDS-R, Nutrition Coordinating Center, Division of Epidemiology (C) 2007 by the Regents of the University of Minnesota); 4) assessing fatty acid composition of erythrocyte membranes at the end of each treatment period to assess compliance to treatment food (Lipomics Technologies, West Sacramento, CA).

\section{Data collection}

Participant's body weight was assessed at baseline and end of all treatments (Tanita TBF 310-GS scale, Tanita Corporation of America Inc., Arlington Heights, IL, USA). Twelve-hour fasting blood samples were collected at baseline and end of each treatment period. Serum was separated by centrifugation, aliquoted and stored at $-80^{\circ} \mathrm{C}$ until further analyses. The erythrocytes were washed and stored in saline at $-80^{\circ} \mathrm{C}$ until further analyses.

\section{Analyses of serum markers}

Lipomics Technologies (West Sacramento, CA) extracted and analyzed the erythrocyte membrane lipids as described previously [24]. The Nutritional Assessment Core at the University of California, Davis (supported by NIH NIDDK grant 35747), performed the serum lipid and lipoprotein analyses $[14,24]$. Serum interleukin $1 \beta$ (IL-1 $\beta)$, interleukin 6 (IL-6), tumor necrosis factor alpha (TNF- $\alpha$ ), soluble intracellular adhesion molecule-1 (sICAM-1), and sE-Selectin were analyzed utilizing the quantitative high sensitivity technique according to the supplier's instructions (R\&D Systems, Minneapolis, MN). High-sensitivity C-reactive protein (Hs-CRP) was analyzed using Enzymelinked immunosorbent assay (Diagnostic Systems Laboratories, Inc., Webster, TX).

\section{Statistical analysis}

Analysis was carried out using the SAS Mixed Procedure (SAS/STAT software, Version 9.2 of the SAS/SAT System for Unix, 2008. SAS Institute Inc., Cary, NC), which correctly analyzes data with heterogeneous variances. A heterogeneity model was used for arachidonic acid and total n-3 fatty acids, and homogeneity model was used for all other nutrients. When ANOVA was significant $(\mathrm{P}<0.05)$, paired comparison tests among the treatments were carried out, and adjusted for multiple comparisons by using the Tukey-Cramer method. Results are presented as least-squares means and 95\% CIs after back-transformation.

Erythrocyte membrane fatty acids, blood lipids and inflammatory factors data were analyzed by using mixed linear models that included fixed terms for treatment and period, and a random term for subjects. TAG, HDL-C and all inflammatory factors except sICAM, were normalized by log-transformation. Results for these outcomes were transformed back into their original units, and are presented as least-squares geometric means and their $95 \%$ C.I. Results with a $P$ value of $<0.05$ were considered statistically significant.

Dietary EPA and DHA, which contained many zerovalued observations, could not be normalized by transformation. Therefore, tests for significant differences among treatments were carried out by using two-way, fixed-effects ANOVA on the ranks of the subject means. When ANOVA was significant, paired comparison tests were used, and adjusted for multiple comparisons.

Baseline values for the variables are not shown in tables as this is a crossover study design where the comparison between each treatment is more relevant than comparison to baseline; however, the baseline-adjusted analyses was carried out utilizing fixed covariates representing the period baseline and the subject-average period baselines were added to the mixed-effects model for serum lipids, inflammatory markers and erythrocyte membrane fatty acid analysis.

\section{Results and discussion Subjects}

Six participants withdrew from the study because of family or job pressures or allergies unrelated to treatment foods. Twenty participants completed the study. The average subject age was $38 \pm 3$ years (range 2164 years) and BMI was $23 \pm 1$. There were 16 females and 4 males representing several ethnic groups: White $(n=10)$, Latino $(n=5)$, Asian $(n=2)$, and other $(n=3)$.

\section{Dietary intake and compliance}

The dietary intakes from the 24-hour recalls for energy, carbohydrate, fiber, total fat, saturated fat, and monounsaturated fat were not significantly different between the three treatments (Table 1). As expected, walnut group had higher intake of total polyunsaturated fat (PUFA), total n-6 and n-3 PUFA, LA, ALA compared to the egg treatment groups since walnut treatment group had 1.5 times higher LA and 3 times higher ALA from diet than the egg treatment groups.

Similarly egg treatment groups had higher protein, arachidonic acid and cholesterol than walnut treatment 
Table 1 Diet composition from the 24-hour recalls*

\begin{tabular}{|c|c|c|c|c|c|c|c|}
\hline \multicolumn{8}{|c|}{ DIET TREATMENTS } \\
\hline \multirow[b]{2}{*}{ Diet variable } & \multicolumn{2}{|c|}{ N-3 FA enriched egg } & \multicolumn{2}{|c|}{ Standard egg (control) } & \multicolumn{3}{|c|}{ Walnut } \\
\hline & $\begin{array}{l}\text { LS mean }{ }^{1} \\
\left(95 \% \mathrm{Cls}^{2}\right)\end{array}$ & $\begin{array}{c}\text { Percent } \\
\text { energy }\end{array}$ & $\begin{array}{l}\text { LS mean } \\
\left(95 \% \mathrm{Cls}^{2}\right)\end{array}$ & $\begin{array}{l}\text { Percent } \\
\text { energy }\end{array}$ & $\begin{array}{l}\text { LS mean } \\
\left(95 \% \mathrm{Cls}^{2}\right)\end{array}$ & $\begin{array}{l}\text { Percent } \\
\text { energy }\end{array}$ & $\begin{array}{c}\text { Global } \\
\text { P-value }^{3}\end{array}$ \\
\hline \multirow{2}{*}{ Kcal } & 1902 & - & 1895 & - & 1729 & - & 0.159 \\
\hline & $(1766,2049)$ & & $(1744,2060)$ & & $(1597,1872)$ & & \\
\hline \multirow{2}{*}{ KJ } & 7963 & - & 7934 & - & 7239 & - & 0.159 \\
\hline & $(7394,8579)$ & & $(7302,8625)$ & & $(6686,7838)$ & & \\
\hline \multirow{2}{*}{ Protein (g) } & $69^{a}$ & 14.51 & $59^{a}$ & 12.45 & $54^{\mathrm{b}}$ & 12.49 & $<0.013$ \\
\hline & $(62,76)$ & & $(53,67)$ & & $(48,61)$ & & \\
\hline \multirow{2}{*}{ Carbohydrate (g) } & 281 & 50.10 & 278 & 58.68 & 248 & 57.37 & 0.172 \\
\hline & $(255,309)$ & & $(250,309)$ & & $(224,275)$ & & \\
\hline \multirow{2}{*}{ Total fat (g) } & 58 & 27.4 & 58 & 27.6 & 64 & 33.3 & 0.537 \\
\hline & $(50,67)$ & & $(50,68)$ & & $(55,75)$ & & \\
\hline \multirow{2}{*}{ Saturated (g) } & 16.3 & 7.71 & 15.9 & 7.55 & 15.1 & 7.86 & 0.8138 \\
\hline & $(13.7,19.4)$ & & $(13.1,19.4)$ & & $(12.5,18.1)$ & & \\
\hline \multirow{2}{*}{ MUFA (g) } & 21.9 & 10.4 & 21.8 & 10.4 & 20.1 & 10.5 & 0.7558 \\
\hline & $(18.4,26.1)$ & & $(17.9,26.4)$ & & $(16.6,24.2)$ & & \\
\hline \multirow{2}{*}{ PUFA (g) } & $13.8^{\mathrm{a}}$ & 6.53 & $14.7^{\mathrm{a}}$ & 6.98 & $22.5^{\mathrm{b}}$ & 11.7 & $<0.0001$ \\
\hline & $(11.8,16.1)$ & & $(12.3,17.5)$ & & $(19.0,26.6)$ & & \\
\hline \multirow{2}{*}{ Total n-6 (g) } & $11.0^{\mathrm{a}}$ & 5.21 & $12.3^{\mathrm{a}}$ & 5.84 & $18.9^{\mathrm{b}}$ & 9.84 & $<0.0001$ \\
\hline & $(9.4,12.9)$ & & $(10.3,14.6)$ & & $(16.0,22.3)$ & & \\
\hline \multirow{2}{*}{ Linoleic (g) } & $10.9^{\mathrm{a}}$ & 5.16 & $12.2^{\mathrm{a}}$ & 5.79 & $18.9^{b}$ & 9.84 & $<0.0001$ \\
\hline & $(9.3,12.7)$ & & $(10.3,14.5)$ & & $(16.0,22.3)$ & & \\
\hline \multirow{2}{*}{ Arachidonic (g) } & $1.14^{\mathrm{a}}$ & 0.54 & $1.05^{\mathrm{b}}$ & 0.50 & $1.01^{\mathrm{c}}$ & 0.53 & $<0.0001$ \\
\hline & $(1.09,1.18)$ & & $(1.04,1.07)$ & & $(1.00,1.02)$ & & \\
\hline \multirow{2}{*}{ Total n-3 (g) } & $1.16^{\mathrm{a}}$ & 0.55 & $1.38^{\mathrm{a}}$ & 0.66 & $3.10^{\mathrm{b}}$ & 1.61 & $<0.0001$ \\
\hline & $(0.93,1.44)$ & & $(1.08,1.75)$ & & $(2.65,3.63)$ & & \\
\hline \multirow{2}{*}{ a-Linolenic (g) } & $1.04^{\mathrm{a}}$ & 0.49 & $1.35^{\mathrm{a}}$ & 0.64 & $3.07^{\mathrm{b}}$ & 1.61 & $<0.0001$ \\
\hline & $(0.86,1.26)$ & & $(1.09,1.67)$ & & $(2.50,3.76)$ & & \\
\hline \multirow{2}{*}{ EPA (g) } & $0.128^{\mathrm{a}}$ & 0.06 & $0.003^{b}$ & 0.00 & $0.001^{b}$ & 0.00 & $<0.0001$ \\
\hline & $(0,0.676)$ & & $(0,0.020)$ & & $(0,0.015)$ & & \\
\hline \multirow{2}{*}{ DHA (g) } & $0.126^{a}$ & 0.06 & $0.021^{b}$ & 0.00 & $0.002^{c}$ & 0.00 & $<0.0001$ \\
\hline & $(0,0.378)$ & & $(0,0.080)$ & & $(0,0.015)$ & & \\
\hline \multirow{2}{*}{ Cholesterol (mg) } & $214^{\mathrm{a}}$ & - & $136^{\mathrm{a}}$ & - & $46^{\mathrm{b}}$ & - & $<0.0002$ \\
\hline & $(131,351)$ & & $(78,236)$ & & $(27,77)$ & & \\
\hline \multirow{2}{*}{ Fiber (g) } & 25 & - & 28 & - & 25 & - & 0.458 \\
\hline & $(22,29)$ & & $(24,32)$ & & $(21,28)$ & & \\
\hline
\end{tabular}

\footnotetext{
${ }^{*}$ Means of three 24-hour diet recalls.
}

${ }^{1}$ Least squares means (LS Means), after back transformation, from 2-way ANOVA with fixed effects for treatment and subject performed on log-transformed data. For EPA and DHA, the recalls for each subject were averaged, and the mean of these subject means is presented. Values in the same row with different superscript letters are significantly different $p<0.05$.

${ }^{2}$ Ranges, instead of $95 \% \mathrm{Cls}$, are given for EPA and DHA.

${ }^{3}$ P-values respectively for N-3 fatty acid enriched egg and standard egg, N-3 fatty acid enriched egg vs. walnut, and standard egg vs. walnut, adjusted for multiple comparisons by the Tukey-Cramer method; except for EPA and DHA which were adjusted by using the Conover method.

group. In addition the n-3 FA enriched egg treatment group had higher EPA and DHA than the other two treatment groups and the standard egg treatment had higher DHA than the walnut treatment group which more or less reflects the fatty acid composition of the treatment food. The higher ALA in standard egg group 
compared to $\mathrm{n}-3$ fatty acid enriched group although not significant could reflect other sources of ALA in the diet and not the treatment per se.

Mixed linear model analyses for changes in subject body weight and body composition were not significant (data not shown), indicating that participants maintained energy balance even after adding a treatment food to their habitual diets. The body composition data established confidence in the 24-hour recall information since calorie intake and weight change was not significantly different during each treatment.

Incorporation of fatty acids into the erythrocyte membrane is presented in Table 2. As expected, LA and ALA were significantly higher during the walnut compared to the egg treatments. DHA was higher during the $n-3$ fatty acid enriched egg compared to the standard egg and walnut treatment periods. EPA was not significantly different between treatments. There was also a slight decrease in monounsaturated fat in the walnut diet compared to the egg treatments.

\section{Serum cardiovascular disease risk markers}

The serum lipid and lipoprotein values following the three treatments are presented in Table 3. On the walnut treatment there was a significant decrease in total cholesterol, triglyceride and apo B, compared to the standard egg but not the n-3 fatty acid enriched egg treatment. However the total-C: HDL-C ratio was lower in the walnut treatment compared to both the egg treatments. There were no significant differences $(\mathrm{p}>0.05)$ among treatments in any of the inflammatory markers assessed in this study (Table 4).

There is substantial evidence that eating fatty fish at least twice a week offers cardiovascular protection [1-3]. However, for vegetarians and for those that do not include fish in their diet for a variety of reasons, other

Table 2 Fatty acid composition of erythrocyte membranes before and after each of the three dietary treatments

\begin{tabular}{|c|c|c|c|c|c|c|c|}
\hline \multicolumn{8}{|c|}{ DIET TREATMENTS } \\
\hline \multirow{2}{*}{$\begin{array}{l}\text { Fatty acid }{ }^{1} \\
\mu \mathrm{g} / \mathrm{mole}^{2} \%\end{array}$} & \multicolumn{2}{|c|}{ Standard egg (control) } & \multicolumn{2}{|c|}{$\mathrm{N}-3$ fatty acid enriched egg } & \multicolumn{2}{|c|}{ Walnut } & \multirow{2}{*}{$\begin{array}{c}\text { Global } \\
P \text { value }^{3}\end{array}$} \\
\hline & Baseline & End $^{2}$ & Baseline & End $^{2}$ & Baseline & End $^{2}$ & \\
\hline \multirow{2}{*}{ Saturated } & 41.1 & 41.0 & 40.9 & 40.9 & 41.4 & 41.2 & 0.9579 \\
\hline & $(40.7,41.5)$ & $(40.2,41.9)$ & $(40.5,41.3)$ & $(39.7,42.1)$ & $(40.8,42.0)$ & $(40.0,42.3)$ & \\
\hline \multirow{2}{*}{ Trans $^{4}$} & 0.05 & 0.02 & 0.06 & 0.03 & 0.06 & 0.03 & 0.5076 \\
\hline & $(0.04,0.06)$ & $(0.01,0.03)$ & $(0.04,0.08)$ & $(0.02,0.06)$ & $(0.05,0.07)$ & $(0.02,0.05)$ & \\
\hline \multirow{2}{*}{ Monounsaturated } & 15.1 & 15.3 & 15.4 & 15.4 & 14.9 & 14.4 & $<0.0003$ \\
\hline & $(14.8,15.4)$ & $(14.8,15.7)^{a}$ & $(15.2,15.7)$ & $(15.0,15.9)^{a}$ & $(15.2,14.6)$ & $(13.9,14.8)^{b}$ & \\
\hline \multirow{2}{*}{ Polyunsaturated } & 36.3 & 35.6 & 35.6 & 35.8 & 36.1 & 36.0 & 0.1761 \\
\hline & $(35.9,36.7)$ & $(34.8,36.4)$ & $(35.3,35.9)$ & $(35.0,36.7)$ & $(34.5,36.5)$ & $(35.8,37.4)$ & \\
\hline \multirow{2}{*}{ n-6 (total) } & 30.4 & 29.7 & 29.8 & 29.7 & 30.0 & 30.5 & 0.2880 \\
\hline & $(30.1,30.7)$ & $(29.1,30.4)$ & $(29.4,30.2)$ & $(28.7,30.6)$ & $(29.6,30.4)$ & $(29.6,31.5)$ & \\
\hline \multirow{2}{*}{ Linoleic } & 11.5 & 10.9 & 11.3 & 10.8 & 11.4 & 11.9 & $<0.0001$ \\
\hline & $(11.3,11.7)$ & $(10.5,11.3)^{a}$ & $(11.0,11.6)$ & $(10.4,11.3)^{a}$ & $(11.1,11.7)$ & $(11.5,12.3)^{b}$ & \\
\hline \multirow{2}{*}{ Arachidonic } & 13.6 & 13.4 & 13.2 & 13.6 & 13.3 & 13.4 & 0.7111 \\
\hline & $(13.3,13.9)$ & $(13.0,13.7)$ & $(12.9,13.5)$ & $(13.2,13.9)$ & $(13.02,13.6)$ & $(13.0,13.7)$ & \\
\hline \multirow{2}{*}{ n-3 (total) } & 5.7 & 5.68 & 5.6 & 5.97 & 6.0 & 5.79 & 0.0903 \\
\hline & $(5.5,5.9)$ & $(5.48,5.88)$ & $(5.4,5.8)$ & $(5.76,6.17)$ & $(5.76,6.24)$ & $(5.59,5.98)$ & \\
\hline \multirow{2}{*}{ a-Linolenic } & 0.14 & 0.14 & 0.13 & 0.15 & 0.18 & 0.19 & $<0.0000$ \\
\hline & $(0.13,0.15)$ & $(0.12,0.16)^{a}$ & $(0.12,0.14)$ & $(0.13,0.16)^{a}$ & $(0.17,0.19)$ & $(0.18,0.20)^{b}$ & \\
\hline \multirow{2}{*}{$E P A^{5}$} & 0.28 & 0.34 & 0.31 & 0.30 & 0.30 & 0.31 & 0.1824 \\
\hline & $(0.25,0.31)$ & $(0.31,0.38)$ & $(0.29,0.33)$ & $(0.26,0.33)$ & $(0.27,0.33)$ & $(0.27,0.34)$ & \\
\hline \multirow{2}{*}{$\mathrm{DHA}^{5}$} & 2.79 & 2.90 & 2.72 & 3.15 & 3.12 & 2.71 & $<0.0015$ \\
\hline & $(2.64,2.94)$ & $(2.74,3.05)^{b}$ & $(2.57,2.87)$ & $(3.00,3.32)^{a}$ & $(2.90,3.34)$ & $(2.55,2.87)^{b}$ & \\
\hline
\end{tabular}

${ }^{1} \mathrm{~N}=18$. Fatty acid values are presented in least square means with $95 \%$ confidence intervals. Values within a row with different superscript letters are significantly different, $P<0.05$.

${ }^{2}$ End values are baseline-adjusted according to the method of Kenward and Roger.

${ }^{3}$ Mixed effects model, controlling for treatment and period effect, adjusted for multiple comparisons using Tukey-Kramer.

${ }^{4}$ Analysis was performed after log-transformation. Results were transformed back into original units and are presented as geometric mean and $\mathrm{Cl}$.

${ }^{5} \mathrm{EPA}=$ eicosapentaenoic acid; $\mathrm{DHA}=$ docosahexaenoic acid. 
Table 3 Serum lipids and lipoproteins before and after each of the three dietary treatments

\begin{tabular}{|c|c|c|c|c|c|c|c|}
\hline \multicolumn{8}{|c|}{ DIET TREATMENTS } \\
\hline \multirow[b]{2}{*}{ Lipid variable $^{1}$} & \multicolumn{2}{|c|}{ Standard egg (control) } & \multicolumn{2}{|c|}{ n-3 FA enriched egg } & \multicolumn{2}{|c|}{ Walnut } & \multirow{2}{*}{$\begin{array}{c}\text { Global } \\
P \text { Value }^{3}\end{array}$} \\
\hline & Baseline & $\mathrm{End}^{2}$ & Baseline & End & Baseline & End & \\
\hline \multicolumn{8}{|l|}{ Cholesterol(s) } \\
\hline Totrol mol & 4.79 & 5.09 & 4.62 & 4.96 & 4.79 & 4.77 & \multirow{2}{*}{$<0.0348$} \\
\hline lotal (mmol/L) & $(4.67,4.91)$ & $(4.92,5.26)^{\mathrm{a}}$ & $(4.50,4.74)$ & $(4.79,5.12)^{\mathrm{ab}}$ & $(4.67,4.92)$ & $(4.60,4.94)^{b}$ & \\
\hline \multirow{2}{*}{$\mathrm{HDL}^{4}(\mathrm{mmol} / \mathrm{L})$} & 1.29 & 1.33 & 1.27 & 1.30 & 1.28 & 1.31 & \multirow{2}{*}{0.7736} \\
\hline & $(1.17,1.41)$ & $(1.25,1.41)$ & $(1.15,1.40)$ & $(1.23,1.38)$ & $(1.15,1.40)$ & $(1.23,1.39)$ & \\
\hline \multirow{2}{*}{ LDL (mmol/L) } & 2.75 & 3.03 & 2.63 & 2.96 & 2.81 & 2.86 & \multirow{2}{*}{0.2866} \\
\hline & $(2.63,2.87)$ & $(2.87,3.19)$ & $(2.50,2.75)$ & $(2.80,3.12)$ & $(2.69,2.94)$ & $(2.70,3.02)$ & \\
\hline \multirow{2}{*}{ LDL:HDL } & 2.13 & 2.31 & 2.06 & 2.32 & 2.20 & 2.19 & \multirow{2}{*}{0.1432} \\
\hline & $(2.03,2.25)$ & $(2.19,2.43)$ & $(1.97,2.18)$ & $(2.20,2.44)$ & $(2.00,2.40)$ & $(2.07,2.31)$ & \\
\hline \multirow{2}{*}{ Total cholesterol:HDL } & 3.71 & 3.86 & 3.63 & 3.84 & 3.76 & 3.64 & \multirow{2}{*}{$<0.013$} \\
\hline & $(3.48,4.00)$ & $(3.72,4.00)^{\mathrm{a}}$ & $(3.40,3.91)$ & $(3.70,3.98)^{a}$ & $(3.51,4.05)$ & $(3.50,3.79)^{b}$ & \\
\hline \multirow{2}{*}{ Triacylglycerides $^{4}(\mathrm{mmol} / \mathrm{L})$} & 1.15 & 1.12 & 1.13 & 0.97 & 1.13 & 0.92 & \multirow{2}{*}{$<0.0434$} \\
\hline & $(1.10,1.21)$ & $(1.00,1.26)^{a}$ & $(1.07,1.18)$ & $(0.87,1.08)^{\mathrm{ab}}$ & $(1.07,1.18)$ & $(0.83,1.03)^{b}$ & \\
\hline \multirow{2}{*}{ Apolipoprotein A (g/L) } & 1.54 & 1.62 & 1.56 & 1.59 & 1.57 & 1.59 & \multirow{2}{*}{0.6416} \\
\hline & $(1.49,1.59)$ & $(1.57,1.67)$ & $(1.51,1.61)$ & $(1.53,1.64)$ & $(1.53,1.62)$ & $(1.54,1.64)$ & \\
\hline \multirow{2}{*}{ Apolipoprotein B (g/L) } & 0.84 & 0.94 & 0.82 & 0.89 & 0.89 & 0.86 & \multirow{2}{*}{$<0.0211$} \\
\hline & $(0.77,0.91)$ & $(0.90,0.98)^{\mathrm{a}}$ & $(0.77,0.87)$ & $(0.85,0.93)^{\mathrm{ab}}$ & $(0.82,0.96)$ & $(0.82,0.90)^{b}$ & \\
\hline \multirow{2}{*}{ Triacylglycerides: Apo $B^{4}$} & 1.37 & 1.10 & 1.38 & 1.02 & 1.27 & 1.01 & \multirow{2}{*}{0.4541} \\
\hline & $(1.33,1.43)$ & $(0.99,1.22)$ & $(1.36,1.39)$ & $(0.91,1.13)$ & $(1.23,1.31)$ & $(0.91,1.12)$ & \\
\hline \multirow{2}{*}{ Apo B: Apo A } & 0.55 & 0.59 & 0.52 & 0.56 & 0.57 & 0.54 & \multirow{2}{*}{0.0527} \\
\hline & $(0.52,0.57)$ & $(0.56,0.62)$ & $(0.51,0.54)$ & $(0.54,0.59)$ & $(0.54,0.59)$ & $(0.51,0.57)$ & \\
\hline
\end{tabular}

${ }^{1}$ Values within a row with different superscript letters are significantly different, $\mathrm{P}<0.05$. All results are presented as geometric mean and $95 \% \mathrm{Cl}$.

${ }^{2}$ End values are baseline- adjusted according to the method of Kenward and Roger.

${ }^{3}$ Mixed effects model, controlling for treatment and period effect, adjusted for multiple comparisons using Tukey-Kramer.

${ }^{4}$ Analysis performed after log-transformation. Results have been transformed back into original units and presented as geometric mean and $95 \% \mathrm{Cl}$.

dietary sources of long chain n-3 fatty acids EPA and DHA are n-3 fatty acid enriched eggs and foods fortified or enriched with $n-3$ fatty acids $[1,6]$. For vegans it is even more difficult to rely on diet alone to obtain long chain n-3 fatty acids and they may have to resort to supplements made from microalgae oils [9]. Although the body can convert the plant $\mathrm{n}-3$ fatty acid ALA into EPA, the conversion to DHA seems to be less efficient [10]. In this study we compared ALA rich walnuts and n-3 fatty acid enriched (primarily DHA) eggs with a standard egg control with respect to their effects on select CVD risk factors among lacto-ovo-vegetarians (LOV). Adding 24 g/d

Table 4 Serum inflammatory markers after each of the three dietary treatments

\begin{tabular}{|c|c|c|c|c|}
\hline \multicolumn{5}{|c|}{ DIET TREATMENTS } \\
\hline Inflammatory marker & Standard egg (control) & n-3 FA enriched egg & Walnut & Global $P$-value ${ }^{1}$ \\
\hline TNF- $a^{2}(p g / m L)$ & $0.33(0.15,0.72)$ & $0.30(0.14,0.65)$ & $0.32(0.15,0.69)$ & 0.30 \\
\hline E-selectin ${ }^{2}(\mathrm{ng} / \mathrm{mL})$ & $0.79(0.55,1.12)$ & $0.72(0.51,1.02)$ & $0.77(0.55,1.10)$ & 0.43 \\
\hline $\mathrm{IL}-1^{2}(\mathrm{pg} / \mathrm{mL})$ & $3.10(2.52,3.82)$ & $3.19(2.60 .3 .92)$ & $2.99(2.43,3.67)$ & 0.71 \\
\hline $\mathrm{IL}-6^{2}(\mathrm{pg} / \mathrm{mL})$ & $0.89(0.66,1.19)$ & $0.97(0.72,1.29)$ & $0.79(0.59,1.06)$ & 0.23 \\
\hline $\operatorname{sICAM}^{3}(n g / m L)$ & $9.13(8.20,10.06)$ & $9.42(8.79,10.05)$ & $9.78(8.84,10.73)$ & 0.81 \\
\hline hs-CRP $(\mathrm{ng} / \mathrm{mL})$ & $1.95(1.39,2.51)$ & $2.64(1.33,3.96)$ & $2.36(1.31,3.42)$ & 0.75 \\
\hline
\end{tabular}

${ }^{1}$ Global P-value for significant difference among treatments.

${ }^{2} \mathrm{P}$-values were determined by using mixed linear models, adjusting for subject and period effects, on log-transformed data. Results are least square means and 95\% Cls after back-transformation.

${ }^{3} \mathrm{P}$-values determined by using Friedman's chi-squared test.: TNF- $\mathrm{a}$-Tumor necrosis factor- $\mathrm{a}$; IL-Interleukin; sICAM-Soluble intracellular adhesion molecule; hs-CRP-High sensitivity c-reactive protein. 
(1 ounce, $6 \mathrm{~d} /$ week) of walnuts to the habitual diet of LOV lowered total-C and TAG compared to eating 6 standard eggs a week. More importantly, adding ALA rich walnuts to the habitual diet of LOV lowered the ratio of total: HDL cholesterol compared to both egg treatments, even when the egg contained a significant amount of DHA.

Previous studies have shown that feeding 42-57 grams of walnuts per day (1.5-2 ounces/d) lowers total-C, LDL$\mathrm{C}$ and TAG in both normal and hypercholesterolemic individuals [21,22,25-28]. While we showed a significant lowering in total-C and TAG, LDL-C remained unchanged. This could either be due to lower baseline LDL-C levels in our normocholesterolemic subjects or because of the low dose of walnuts (' $24 \mathrm{~g} / \mathrm{d}$ ) used in our study compared to higher amounts typically used in other feeding studies [14,21,22,25-27]. The lowering of serum total cholesterol by walnuts is consistent with previous findings $(13,20)$. While it seems that this is due to a lower intake of cholesterol in the walnut treatment compared to the egg treatments, it is known that dietary fatty acids are better predictors of serum cholesterol than dietary cholesterol per se. In fact we have observed decreases in serum cholesterol following walnut enriched diet even when dietary cholesterol intake was similar to the control diet (20).

In previous studies it has been observed that DHA increases LDL-C $[14,29]$. It was interesting however; that the n-3 fatty acid enriched egg treatment resulted in cholesterol levels lower than the standard egg treatment although the values were not significant statistically. This may be attributed to the higher ALA content $(1 \mathrm{~g} /$ egg yolk) of the n-3 fatty acid enriched eggs (almost half of what was found in the one ounce of walnuts) which were derived from flaxseed meal fed chicken compared to only $0.1 \mathrm{~g}$ ALA per standard egg yolk.

In a previous study comparing fatty fish and walnuts, we showed that walnuts lowered total and LDL-C, while the fatty fish lowered TAG and increased HDL-C compared to the other treatment [14]. In the present study, TAG decreased 13\% (not statistically significant) on the n-3 fatty acid enriched egg treatment and $18 \%$ on the walnut treatment compared to the standard egg treatment. $13-18 \%$ decrease is clinically relevant and suggests that ALA and EPA/DHA can both lower triglyceride levels. In our previous study with fatty fish and walnuts we showed a two fold increase in erythrocyte EPA levels following fatty fish intake compared to walnuts and a corresponding lowering of TAG only in the fatty fish diet group [14]. In this study the EPA in erythrocyte membrane were not different among treatments but DHA was significantly higher in the $\mathrm{n}-3$ fatty acid enriched egg treatment compared to the other two treatments. These observations do not help explain the greater TAG lowering seen with walnut treatment. However, the total $n-3$ fatty acids derived from the diet was 3 times higher in the walnut treatment compared to the egg treatments. Perhaps this may explain why walnuts produced a more significant effect on TAG than the n-3 fatty acid enriched eggs.

The total: HDL cholesterol ratio is considered a clinical benchmark to assess risk of CVD [30]. Walnut treatment was most effective in reducing this ratio compared to both the egg treatments. Along with this ratio, the Apo B: Apo A-1 ratio also approached significant difference between treatments with a lower ratio after the walnut treatment compared to the standard egg treatment. This suggests that overall a more favorable lipid profile was achieved through eating 1 ounce of walnuts 6 times a week compared to eating 6 standard eggs.

These effects of walnuts may be mediated partly by the fatty acid composition of walnuts but may extend beyond the lipid matrix of the walnut. The EPA levels in erythrocyte membrane are similar in all three treatments. Although EPA levels in the walnut treatment group did not increase as expected, this might be due to the high n-6 fatty acid intake. On the other hand the DHA level in erythrocyte membrane in the walnut treatment was significantly lower than the n-3 FA enriched egg treatment. This suggests that consumption of ALA rich foods alone may not be sufficient to achieve DHA status in LOVs when compared to LOVs that consume a direct source of DHA. Future research looking at a higher dose of walnuts or other ALA rich foods, or a diet treatment longer than 8 weeks may be required to determine if erythrocyte incorporation of DHA increases under these conditions. However for the cardiovascular risk markers measured, the levels of DHA in erythrocyte membrane does not seems to be a limiting factor, at least in this study.

Inflammation plays a role in the development and progression of atherosclerosis and contributes to an increased risk of CVD [7]. There is some evidence to suggest that $\mathrm{n}-3$ fatty acids may lower inflammation $[7,8,22]$, as indicated by serum markers of inflammation, but these finding are not consistent [31,32]. In our study we did not show any significant changes in inflammatory markers following either walnut or $\mathrm{n}-3$ fatty acid enriched egg treatments. Most of the studies that have looked at healthy individuals and found no effect of $n-3$ fatty acids on inflammatory biomarkers especially the cytokines [31]. However, the soluble adhesion molecules sICAM-1 and sVCAM-1 were decreased following intake of n-3 fatty acids in healthy subjects but only at high dose ( 2 g/d of EPA and DHA) or with longer duration of intervention (12 weeks) [32]. Subjects in our study on the $\mathrm{n}-3$ fatty acid enriched eggs obtained $\sim 500 \mathrm{mg}$ DHA per egg yolk but very little EPA ( 30 mg/egg yolk) 
and for only 8 weeks. A similar beneficial effect of walnuts on the adhesion molecule sE-selectin has been observed, but only when walnuts and other ALA rich foods contributed at least $6.5-12 \%$ of the total energy of the diet [33-35]. Reducing levels of cellular adhesion molecules will have a significant role in modulating the atherosclerotic process and therefore, future studies considering dietary modification with n-3 fatty acids must be mindful of appropriate dose and duration especially, among healthy individuals.

Previously we observed that plasma levels of eicosanoids such as prostaglandin $\left(\mathrm{PGE}_{2}\right)$ and thromboxane $\left(\mathrm{TXA}_{2}\right)$ metabolites were lowered following the intake of two fatty fish meals (EPA + DHA of $780 \mathrm{mg} / \mathrm{d}$ ) per week [34]. It is known that the eicosanoids produced from EPA is anti-inflammatory and those derived from arachidonic acid pro-inflammatory [36]. The effect that we observed was also seen with walnut intake $(42.5 \mathrm{~g} / \mathrm{d})$ in that same study, which is attributed to not just the ALA content but also to the $\gamma$-tocopherol content of walnuts, known to inhibit the activity of cyclooxygenase enzyme [37]. Since we did not measure these biomarkers in the present study, whether or not these markers would have been influenced following the consumption of low dose walnuts or n-3 fatty acid enriched eggs remains unresolved.

This was a free-living study with the rigor of control not nearly the same as in metabolically controlled studies. On the other hand, free living conditions in which subjects follow their habitual diet with very little dietary modification provides more generalizable results since it reflects more realistically what the general population would eat. Since this study was done in healthy LOVs who limit the amount of animal products in their diet, caution must be used in interpretation of the results for people with chronic diseases, people that consume other animal products and people that may exhibit sensitivity to dietary cholesterol.

\section{Conclusion}

Our study suggests that for lacto-ovo-vegetarians, replacing standard eggs with n-3 fatty acid enriched eggs in their diet may be an option to increase DHA content of erythrocyte membranes. However, to influence blood lipid risk factors, it seems that eating a handful of walnuts daily may have overall better benefits for lactoovo-vegetarians.

\footnotetext{
Abbreviations

ALA: a-linolenic acid; CVD: Cardiovascular disease; DHA: Docosahexaenoic acid; EPA: Eicosapentaenoic; FA: Fatty acids; HDL-C: High density lipoprotein cholesterol; Hs-CRP: High- sensitivity C-reactive protein; IL-1B: Interleukin 1B; IL-6: Interleukin 6; LOV: Lacto-ovo-vegetarians; LDL-C: Low density lipoprotein cholesterol; FA: n-3 Fatty acids n-3; PGE : Prostaglandin 2; RD: Registered dietitian; sICAM-1: Soluble intracellular adhesion molecule-1; Total-C: Total cholesterol; TXA 2 : Thromboxane 2; TAG: Triacylglycerol; TNF-a: Tumor necrosis factor alpha.
}

\section{Competing interests}

There are no conflicts of interest. This work was supported by the American Egg Board Fellowship; Agriculture Research Institute (Grant) from California State Polytechnic University, Pomona; California Walnut Commission (in-kind donation of walnuts); Chino Valley Ranchers (in-kind donation of eggs). Several authors (JS, EH, SR) have received previous research grants from the walnut and other nut industries. BBW received a dissertation fellowship grant from the American Egg Board, and a grant from the Agriculture Research Institute, both of which were used entirely to fund this study. BBW received a prior grant from the disbanded California Egg Commission to compile recent egg-related research in 1999.

\section{Authors' contributions}

BBW was responsible for the study conception and design, subject recruitment and selection, interpretation of the data, management of the feeding trial, drafting of the manuscript, and obtaining the funding for the study. EH provided lab support. SR helped with study design, performed subject recruitment and selection, interpreted the data, reviewed and edited the manuscript. EH and JS helped develop the study design, assisted in data interpretation and reviewed the manuscript. All authors read and approved the final manuscript.

\section{Acknowledgments}

We would like to thank the study participants, Chino Valley Ranchers for the egg donations, and the California Walnut Commission for donating the study walnuts. We wish to thank Dr. Karen Jaceldo-Siegl, faculty in the Department of Nutrition, Loma Linda University for performing the dietary analysis, and Dr. Suwimol Sapwarobol, Faculty of Allied Health Sciences, Chulalongkorn University, Bangkok, Thailand, for performing all immunological assays, and Jay Tanzman for performing the statistical analyses.

\section{Author details}

${ }^{1}$ Department of Nutrition, School of Public Health, Loma Linda University, Loma Linda, CA 92350, USA. ²Department of Human Nutrition and Food Science, California State Polytechnic University, Pomona, 3801 West Temple Ave, Pomona, CA 91768, USA.

Received: 27 September 2013 Accepted: 18 March 2014 Published: 27 March 2014

\section{References}

1. Gebauer SK, Psota TL, Harris WS, Kris-Etherton PM: N-3 fatty acid dietary recommendations and food sources to achieve essentiality and cardiovascular benefits. Am J Clin Nutr 2006, 83(Suppl 6):1526-1535.

2. Kris-Etherton PM, Taylor DS, Yu-Poth S, Huth P, Moriarty K, Fishell V, Hargrove RL, Zhao G, Etherton TD: Polyunsaturated fatty acids in the food chain in the United States. Am J Clin Nutr 2000, 71(Supp 1):179-188.

3. Kris-Etherton PM, Harris WS, Appel L: Fish consumption, fish oil, omega-3 fatty acids, and cardiovascular disease. Circulation 2002, 106:2747-2757.

4. Pan A, Chen M, Chowdhury R, Wu JH, Sun Q, Campos H, Mozaffarian D, Hu FB: a-Linolenic acid and risk of cardiovascular disease: a systematic review and meta-analysis. Am J Clin Nutr 2012, 96:1262-1273.

5. Welch AA, Shakya-Shrestha S, Lentjes MA, Wareham NJ, Khaw KT: Dietary intake and status of $n-3$ polyunsaturated fatty acids in a population of fish-eating and non-fish-eating meat eaters, vegetarians, and vegans and the precursor-product ratio of a-linolenic acid to long chain n-3 polyunsaturated fatty acids: results from the EPIC-Norfolk cohort. Am J Clin Nutr 2010, 92:1040-1051.

6. Davis BC, Kris-Etherton PM: Achieving optimal essential fatty acid status in vegetarians: current knowledge and practical implications. Am J Clin Nutr 2003, 78(Supp 3):640-646.

7. Rangel-Huerta OD, Aguilera CM, Mesa MD, Gil A: Omega-3 long chain polyunsaturated fatty acids supplementation on inflammatory biomarkers: a systematic review of randomized clinical trial. Br J Nutr 2012, 107(Supp 2):159-170.

8. Darshan S, Kelley S, Adkins Y: Similarities and differences between the effects of EPA and DHA on markers of atherosclerosis in human subjects. Proc Nutr Soc 2012, 71:322-331.

9. Conquer JA, Holub BJ: Supplementation with an algae source of docosahexaenoic acid increases ( $n-3)$ fatty acid status and alters 
selected risk factors for heart disease in vegetarian subjects. J Nutr 1996, 126:3032-3039.

10. Brenna JT: Efficiency of conversion of alpha-linolenic acid to long chain n-3 fatty acids in man. Curr Opin Clin Nutr Metab Care 2002, 5:127-132.

11. Erkkila AT, Lichtenstein AH, Mozaffarian D, Herrington DM: Fish intake is associated with a reduced progression of coronary artery atherosclerosis in postmenopausal women with coronary artery disease. Am J Clin Nutr 2004, 80:626-632.

12. Li Z, Lamon-Fava S, Otvos J, Lichtenstein AH, Velez-Carrasco W, McNamara $J R$, Ordovas JM, Schaefer EJ: Fish consumption shifts lipoprotein subfractions to a less atherogenic pattern in humans. J Nutr 2004 13:1724-1728

13. Bulliyya G: Influence of fish consumption on the distribution of serum cholesterol in lipoprotein fractions: comparative study among fish-consuming and non-fish-consuming populations. Asia Pac J Clin Nutr 2002, 1:104-111.

14. Rajaram S, Hasso Haddad E, Mejia A, Sabaté J: Walnuts and fatty fish influence different serum lipid fractions in normal to mildly hyperlipidemic individuals: a randomized controlled study. Am J Clin Nutr 2009, 8:1657S-1663S

15. Bean LD, Leeson S: Long-term effects of feeding flaxseed on performance and egg fatty acid composition of brown and white hens. Poult Sci 2003, 8:388-394.

16. Farrell DJ: Enrichment of hen eggs with $n-3$ long-chain fatty acids and evaluation of enriched eggs in humans. Am J Clin Nutr 1998, 688:538-544.

17. Maki KC, Van Elswyk ME, McCarthy D, Hess SP, Veith PE, Bell M, Subbaiah P, Davidson $\mathrm{MH}$ : Lipid responses in mildly hypertriglyceridemic men and women to consumption of docosahexaenoic acid-enriched eggs. Int Vitam Nutr Res 2003, 73:357-368.

18. Ferrier LK, Caston LJ, Leeson S, Squires J, Weaver BJ, Holub BJ: Alphalinoleic acid and docosahexaenoic acid-enriched eggs from hens fed flaxseed: influence on blood lipids and platelet phospholipids and platelets phospholipid fatty acids in humans. Am J Clin Nutr 1995 62:81-86

19. Surai PF, MacPherson A, Speake BK, Sparks NH: Designer egg evaluation in a controlled trial. Eur J of Clin Nutr 2000, 54:298-305.

20. Lewis NM, Schalch K, Scheideler SE: Serum lipid response to $n-3$ fatty acid enriched eggs in persons with hypercholesterolemia. J Am Dietetic Assoc 2000, 100:365-367

21. Sabaté J, Fraser GE, Burke K, Knutsen SF, Bennett H, Lindsted KD: Effects of walnuts on serum lipid levels and blood pressure in normal men. $N$ Engl J Med 1993, 328:603-607.

22. Zambón D, Sabaté J, Muñoz S, Campero B, Casals E, Merlos M, Laguna JC, Ros E: Substituting walnuts for monounsaturated fat improves the serum lipid profile of hypercholesterolemic men and women. A randomized crossover trial. Ann Intern Med 2000, 132:538-546.

23. Burns-Whitmore BL, Haddad EH, Sabaté J, Jaceldo-Siegl K, Tanzman J, Rajaram S: Effects of $n-3$ fatty acid enriched eggs and organic eggs on serum lutein in free-living lacto-ovo-vegetarians. Eur J Clin Nutr 2010, 64:1332-1337

24. Sabaté J, Haddad E, Tanzman JS, Jambazian P, Rajaram S: Serum lipid response to the graduated enrichment of a Step I diet with almonds: a randomized feeding trial. Am J Clin Nutr 2003, 77:1379-1384.

25. Chisholm A, Mann J, Skeaff M, Frampton C, Sutherland W, Duncan A Tiszavari S: A diet rich in walnuts favourably influences plasma fatty acid profile in moderately hyperlipidaemic subjects. Eur J Clin Nutr 1998, $52: 12-16$.

26. Iwamoto M, Imaizumi K, Sato M, Hirooka Y, Sakai K, Takeshita A, Kono M: Serum lipid profiles in Japanese women and men during consumption of walnuts. Eur J Clin Nutr 2002, 56:629-637.

27. Kris-Etherton PM: Walnuts decrease risk of cardiovascular disease: A summary of efficacy and biologic mechanisms. J Nutr 2014, 144:547S-554S.

28. Griel AE, Kris-Etherton PM: Tree nuts and lipid profile: a review of clinical studies. Br J Nutr 2006, 96(Supp 2):68-78.

29. Jacobson TA, Glickenstein SB, Rowe JD, Soni PN: Effects of eicosapentaenoic acid and docosahexaenoic acid on low density lipoprotein cholesterol and other lipids: a review. J Clin Lipidol 2012, 6:5-18.
30. Nelson GJ, Schmidt PC, Bartolini GL, Kelley DS, Kyle D: The effect of dietary docosahexaenoic acid on plasma lipoproteins and tissue fatty acid composition in humans. Lipids 1997, 32:1137-1146.

31. Myhrstad MC, Retterstøl K, Telle-Hansen VH, Ottestad I, Halvorsen B, Holven $K B$, Ulven SM: Effect of marine $n-3$ fatty acids on circulating inflammatory markers in healthy subjects and subjects with cardiovascular risk factors. Inflamm Res 2011, 60:309-319.

32. Yang Y, Lu N, Chen D, Meng L, Zheng Y, Hui R: Effects of n-3 PUFA supplementation on plasma soluble adhesion molecules: a meta-analysis of randomized controlled trials. Am J Clin Nutr 2012, 95:972-980.

33. Ros E, Núñez I, Pérez-Heras A, Serra M, Gilabert R, Casals E, Deulofeu R: A walnut diet improves endothelial function in hypercholesterolemic subjects: a randomized crossover trial. Circulation 2004, 109:1609-1614.

34. Chiang YL, Haddad E, Rajaram S, Shavlik D, Sabaté J: The effect of dietary walnuts compared to fatty fish on eicosanoids, cytokines, soluble endothelial adhesion molecules and lymphocyte subsets: a randomized, controlled, crossover trial. Prosta Leuko Essent Fatty Acids 2012, 87:111-117.

35. de Mello VDF, Erkkilä AT, Schwab US, Pulkkinen L, Kolehmainen M, Atalay M, Mussalo H, Lankinen M, Oresic M, Lehto S, Uusitupa M: The effect of fatty or lean fish intake on inflammatory gene expression in peripheral blood mononuclear cells of patients with coronary heart disease. Eur J Nutr 2009, 48:447-455

36. Calder PC: The role of marine omega-3 (n-3) fatty acids in inflammatory processes, atherosclerosis and plaque stability. Mol Nutr Food Res 2012 56:1073-1080

37. Jiang Q, Ames BN: Gamma tocopherol, but not alpha-tocophero decreases proinflammatory eicosanoids and inflammation damage in rats. FASEB J 2003, 17:816-822

doi:10.1186/1475-2891-13-29

Cite this article as: Burns-Whitmore et al:: Effects of supplementing n-3 fatty acid enriched eggs and walnuts on cardiovascular disease risk markers in healthy free-living lacto-ovo-vegetarians: a randomized, crossover, free-living intervention study. Nutrition Journal 2014 13:29.

\section{Submit your next manuscript to BioMed Central and take full advantage of:}

- Convenient online submission

- Thorough peer review

- No space constraints or color figure charges

- Immediate publication on acceptance

- Inclusion in PubMed, CAS, Scopus and Google Scholar

- Research which is freely available for redistribution 